\title{
Buscando la inclusión de las minorías en un contexto multicultural. Una revisión teórica del prejuicio y de las estrategias para reducirlo
}

\author{
Miguel Ángel Martín Cárdaba \\ Centro Universitario Villanueva. UCM. Área de Comunicación \\ mmartincar@villanueva.edu
}

\section{Gaspar Brändle}

Universidad de Murcia. Facultad de Economía y Empresa. Departamento de Sociología y Política Social gbrandle@um.es

\section{Resumen}

Asistimos al periodo de mayor movilidad migratoria de la historia y, aunque los motivos de este flujo continuo son diversos (conflictos bélicos, desigualdades geoeconómicas, etc.), una de sus consecuencias más notables es la creciente concentración de grupos minoritarios en algunas sociedades, hasta el punto que llegan a convertirse en un auténtico crisol multicultural. En este contexto, las actitudes que los miembros de dichas sociedades desarrollen hacia tales grupos son claves para facilitar su integración y una convivencia pacífica, armoniosa y beneficiosa para todos. Pero el prejuicio, entendido como una evaluación negativa hacia otro grupo social, es consustancial a cualquier sociedad y especialmente relevante cuando se analizan los conflictos intergrupales. Además, una vez que se han formado, los prejuicios tienden a autoperpetuarse en el tiempo, incluso pueden llegar a ser realmente resistentes al cambio. Por ello, en este trabajo, se pretende abordar dicho problema social a partir de una amplia revisión teórica del prejuicio y de las diferentes propuestas existentes para reducirlo.

Palabras clave: estereotipos; inmigración; discriminación; multiculturalismo; integración social.

Abstract. Seeking the Inclusion of Minorities in a Multicultural Context: A Theoretical Review of Prejudice and Strategies to Reduce It

We are currently witnessing the period of greatest migration movements in history. Although the reasons for this continuing flow of migrants are diverse and include war, geo-economic inequalities and others, one of the most remarkable consequences is the increasing concentration of minority groups in certain societies that become a multiethnic 
melting pot. In this context, the attitudes that members of these societies develop towards such groups are key to facilitating their integration and peaceful, harmonious and mutually beneficial coexistence. However, prejudice, defined as a negative evaluation of another social group, is inherent in all societies and particularly relevant when analyzing intergroup conflict. Furthermore, once formed, prejudice tends to be self-perpetuating throughout time and resistant to change. This paper aims to address this social problem through a theoretical review of prejudice and the different proposals to reduce it.

Keywords: stereotypes; immigration; discrimination; multiculturalism; social integration.

\section{Sumario}

Introducción: la necesidad de superar los prejuicios en las sociedades pluriculturales

La dificultad de combatir el prejuicio: algunos elementos para el análisis
Estrategias de reducción de prejuicio:

soluciones a un problema social

Conclusiones

Referencias bibliográficas

Introducción: la necesidad de superar los prejuicios en las sociedades pluriculturales

Si bien las diferencias raciales, culturales, económicas e, incluso, de estilo de vida son consustanciales a la vida en sociedad, ello no es óbice para que el sentimiento de desconfianza hacia lo que es diferente gobierne parte de las relaciones sociales. En la construcción social que los individuos hacen de la realidad, siempre existe un «nosotros» que se confronta con un «otros», al que con frecuencia, por desconocido, se le ve como una amenaza. Y cuando el grupo de pertenencia se ve ante una amenaza — sea ésta real o infundada-, reacciona cerrándose sobre sí mismo y excluyendo al que es diferente.

Aunque este razonamiento puede simplificar en exceso una realidad ciertamente compleja, permite situarnos ante un problema social de primera magnitud: la exclusión social de aquellos que, por diversos motivos, son considerados diferentes. Es cierto que los grupos considerados diferentes varían de una sociedad a otra y a lo largo del tiempo, pero parece existir un nexo común que elimina las distinciones espacio-temporales: la generación de prejuicios hacia los grupos minoritarios. De hecho, debe ser subrayado que los mecanismos por los que se produce y se perpetúa el prejuicio son comunes y similares en su mayor parte para los diferentes exogrupos (étnicos, religiosos, nacionales, de género, etc.).

No cabe duda de que la generación de prejuicios hacia minorías, y especialmente hacia grupos étnicos y culturales diferentes, es un aspecto de enorme importancia en las sociedades modernas, toda vez que nos situamos en un contexto de enorme movilidad migratoria, tanto en cantidad — recientemente se ha alcanzado un nuevo máximo histórico en el número de migrantes internacionales-, como en extensión — los flujos migratorios se han ido ampliando 
hacia nuevas áreas del planeta- (United Nations, 2009). Este hecho tiene como una de sus principales consecuencias la formación de sociedades extraordinariamente heterogéneas en cuanto a su composición racial, cultural y étnica. España constituye un buen ejemplo en este sentido, ya que acoge a personas originarias de los cinco continentes y que representan a más de 190 nacionalidades diferentes (Observatorio Permanente de la Inmigración, 2012).

Nuestro país ha sido, durante gran parte del siglo XX, un escenario de importantes movimientos migratorios hacia el exterior. Ha presentado saldos migratorios de signo negativo hasta la década de 1970 (Cabré et al., 2002). Pero por diversos motivos que, en todo caso, van más allá del factor económico (Alonso, 2011), la dirección del flujo migratorio se ha invertido en las dos últimas décadas, de modo que se ha convertido en país de recepción de inmigrantes. Tanto es así que, actualmente, ocupa el octavo lugar mundial en recepción de emigración, con 6,4 millones de migrantes internacionales (United Nations, 2009).

Es indudable que las peculiares condiciones y características de nuestro emergente concepto de sociedad, unido a los grandes avances tecnológicos aplicados al campo de las telecomunicaciones y los transportes, permite la aparición de nuevas formas de contacto en un mundo más pequeño e interconectado. Pero, paralelamente, esta creciente multiculturalidad trae consigo unos riesgos y unas evidentes tensiones internas entre grupos de cultura, etnia o religión diferente que exigen de nuestras sociedades una mayor elasticidad y capacidad de adaptación que permitan alcanzar unos niveles mínimos de cohesión social (Solé et al., 2011). Así, además de su impacto evidente sobre la demografía y la economía, esta mayor multiculturalidad puede tener también un impacto indeseable en la sociedad y generar unas percepciones, unas opiniones y unas actitudes negativas (prejuicios) hacia los nuevos colectivos minoritarios que aparecen en nuestras comunidades.

La construcción de esta imagen negativa de la inmigración podría derivarse, en gran medida, de los discursos políticos y de los mensajes que proyectan los medios de comunicación, cuando hacen referencia a la inmigración como un problema que se asocia al incremento de la delincuencia, a la pérdida de la homogeneidad cultural o al deterioro de los servicios y las prestaciones públicas. Evidentemente, todo ello genera el consiguiente efecto pernicioso en la imagen de la inmigración, lo cual colabora a aumentar los prejuicios y los estereotipos contrarios a ella (Cea y Vallés, 2008) e, incluso, contribuye a su estigmatización (Álvarez, 2009). Quizá por ello, cada año aumenta el porcentaje de personas que opinan que el número de inmigrantes es elevado o excesivo $(69 \%$ en 2007, 77\% en 2008 y 79\% en 2009, véase Cea y Vallés, 2010). Fenómeno este que, según algunos autores, podría explicarse por la denominada «psicosis de la invasión» (Vallés et al., 1999).

Debido a su creciente relevancia, diferentes estudios han abordado el fenómeno del prejuicio en España y han mostrado que las actitudes prejuiciosas hacia la inmigración están presentes en mayor o menor medida en nuestra 
Tabla 1. Opiniones hacia la inmigración

\begin{tabular}{lcccccc}
\hline & $\begin{array}{c}\text { Muy de } \\
\text { acuerdo }\end{array}$ & $\begin{array}{c}\text { Más bien } \\
\text { de acuerdo }\end{array}$ & $\begin{array}{c}\text { Más bien en } \\
\text { desacuerdo }\end{array}$ & $\begin{array}{c}\text { Muy en } \\
\text { desacuerdo }\end{array}$ & N. S. N. C. \\
\hline $\begin{array}{l}\text { La presencia de inmigran- } \\
\text { tes hace que disminuya } \\
\text { la calidad de la atención } \\
\text { sanitaria }\end{array}$ & 22,7 & 30,6 & 25,4 & 15,5 & 5,6 & 0,2 \\
$\begin{array}{l}\text { Los inmigrantes abusan } \\
\text { de la atención sanitaria } \\
\text { gratuita }\end{array}$ & 25,7 & 28,6 & 20,3 & 12,3 & 12,5 & 0,7 \\
$\begin{array}{l}\text { Aunque tengan los } \\
\text { mismos ingresos, se les } \\
\text { da más ayudas sanitarias }\end{array}$ & 23,0 & 24,2 & 19,4 & 16,0 & 16,8 & 0,7 \\
$\begin{array}{l}\text { a los inmigrantes que a } \\
\text { los españoles }\end{array}$ & & & & & & \\
$\begin{array}{l}\text { La calidad de la educa- } \\
\text { ción empeora en los cole- } \\
\text { gios donde hay muchos } \\
\text { hijos de inmigrantes }\end{array}$ & 17,9 & 32,2 & 23,8 & 12,6 & 13,0 & 0,6 \\
$\begin{array}{l}\text { Aunque tengan los mis- } \\
\text { mos ingresos, se les da } \\
\text { más ayudas escolares a } \\
\text { los inmigrantes que a los } \\
\text { españoles }\end{array}$ & 26,2 & 25,8 & 15,5 & 12,3 & 19,6 & 0,6 \\
\hline
\end{tabular}

Fuente: Centro de Investigaciones Sociológicas (2009).

sociedad $^{1}$. Desde un enfoque cuantitativo, merece la pena destacar la serie de encuestas llevadas a cabo por el Centro de Investigaciones Sociológicas con el objetivo de ahondar en el conocimiento de las actitudes de los españoles hacia la inmigración (Centro de Investigaciones Sociológicas, 1995, 2008 y 2009). En líneas generales, se podría señalar que los datos muestran una tendencia relativamente sostenida a valorar a los inmigrantes de modo desfavorable, puesto que, en ocasiones, son relacionados con el aumento de la delincuencia y la inseguridad, la apropiación excesiva de los recursos públicos o el deterioro en las condiciones del mercado de trabajo.

A modo de muestra, la tabla 1 podría ayudar a ilustrar esta afirmación, ya que revela un cierto grado de acuerdo por parte de un grupo significativo de la población que sostiene una visión prejuiciosa hacia la inmigración.

Como puede observarse en los datos reflejados en la tabla 1, entre aquellos que tienen una opinión formada en uno u otro sentido (i. e., excluyendo del análisis a los que no saben o no contestan), hay una mayoría cualificada - que llega a alcanzar las dos terceras partes en algunos casos- que asocia la presencia de inmigrantes con el empeoramiento de la calidad de la educación y la

1. Otra muestra de la presencia y la relevancia del prejuicio en España es la creación de nuevos instrumentos de medición, como el de Díez Nicolás (2009), que permite aglutinar los diferentes indicadores utilizados en un único índice de xenofobia y racismo. 
sanidad, les achaca un uso abusivo o inapropiado de estos servicios y les culpa de recibir más ayudas que los españoles.

Por su parte, el Colectivo IOÉ ha hecho lo propio desde una aproximación cualitativa, subrayando que si bien existe un apreciable número de discursos que abogan por la aceptación y la integración, son muchos los discursos prejuiciosos hacia los inmigrantes sustentados en la criminalización y un rechazo visiblemente xenófobo (Colectivo IOÉ, 1995; Colectivo IOÉ y Ortí, 2007).

Ambos enfoques, cuantitativo y cualitativo, han sido también integrados en otra serie de estudios elaborados a instancias del Observatorio Español del Racismo y la Xenofobia (Cea y Vallés, 2009 y 2010), en los que se destaca un carácter bifronte en el imaginario de la sociedad española hacia la inmigración, del que, por un lado, se pueden extraer valoraciones positivas hacia este colectivo, como la riqueza cultural que aportan, pero que, paralelamente, también genera un número aún mayor de manifestaciones que enfatizan aspectos negativos, como los problemas de convivencia o la merma de los servicios públicos por la presencia de inmigrantes.

Como se ha puesto de manifiesto, en prácticamente todos estos estudios se pueden advertir ciertas trazas de prejuicio en el discurso y en algunas de las respuestas de los encuestados, al desprenderse opiniones contrarias e intolerantes hacia los inmigrantes. Los resultados son quizá aun más llamativos si tenemos en cuenta que, cuando se les pregunta directamente a las personas por temas controvertidos (i. e., inmigración), es muy habitual que, por razones de deseabilidad social, oculten o enmascaren sus verdaderas opiniones (Cea, 2009). Y es que, a medida que los valores democráticos de igualdad y de solidaridad van extendiéndose en nuestra sociedad, los prejuicios se convierten en una realidad social menos deseable. Como consecuencia, la gente está cada vez menos dispuesta a reconocer, ante los demás y ante sí misma, que mantiene actitudes prejuiciosas respecto a ciertos grupos sociales. Lo cual, evidentemente, no quiere decir que no los sostenga. En este sentido, Gaertner y Dovidio (1986) pusieron de manifiesto que cada vez es más normal encontrar a personas que tienen una imagen igualitaria y no prejuiciosa de sí mismos y que, sin embargo, se comportan de un modo prejuicioso cuando la estructura normativa es ambigua y/o existen argumentos para racionalizar dicho comportamiento. Este fenómeno ha recibido el nombre de racismo aversivo.

A este respecto, Espelt et al. (2006) llevaron a cabo un estudio en el que pudieron constatar la presencia de racismo aversivo en España. En su estudio, pidieron a un grupo de participantes que previamente habían mostrado una actitud igualitaria y sin traza de prejuicio ante la inmigración, que adoptaran el papel de jurado en un supuesto juicio a un individuo que, dependiendo de la condición experimental, era español o marroquí. Espelt y colaboradores encontraron que, en aquellas circunstancias en las que su conducta podría ser atribuida a una motivación racista (e. g., las pruebas sobre la culpabilidad del acusado eran dudosas), los individuos igualitarios tendían a juzgar de manera igualitaria y equitativa al inmigrante, incluso en ocasiones le favorecían más que cuando se trataba de un español. Sin embargo, en una situación más 
ambigua que les permitía racionalizar con más facilidad una conducta discriminatoria (e. g., existía una prueba concluyente contra el acusado pero el juez ordenaba al jurado no tenerla en consideración por haber sido obtenida mediante procedimientos ilegales), los individuos supuestamente igualitarios tendían a juzgar con más dureza al acusado cuando éste era un inmigrante que cuando el acusado era un español. En definitiva, este estudio es especialmente sugerente, al mostrar que, en determinadas circunstancias, el prejuicio podría hacerse presente incluso entre aquellos sujetos que se consideran libres de él.

Por otro lado, y más allá del análisis de la percepción que tienen los españoles sobre los inmigrantes, diversos estudios han tratado de acercarse a este fenómeno desde la óptica contraria, esto es, a partir de la exploración de las percepciones que los propios inmigrantes tienen sobre las actitudes de la población nativa en relación con ellos. En este sentido, a partir de datos de encuesta, Morales et al. (2008) subrayan que una parte considerable de inmigrantes se sienten discriminados por los prejuicios y las actitudes que los españoles autóctonos manifiestan hacia ellos. Resultados similares son los que alcanza el Colectivo IOÉ (2010) a partir de una exploración cualitativa, donde se recalca que, entre los discursos generados entre los propios inmigrantes, sobresalen algunos que dejan entrever la percepción de un trato discriminatorio, segregador y excluyente por parte de los españoles, especialmente destacado por aquellos que están «sin papeles» o cuyas costumbres y cultura están más alejadas de las dominantes en la sociedad española.

Así pues, en un contexto en el que la evolución de nuestras sociedades está dando lugar a la aparición de nuevas y más complejas formas de discriminación y prejuicio, parece necesario afirmar la especial relevancia del problema y, por tanto, la apremiante oportunidad de su estudio. Por ello, el presente trabajo constituye una revisión teórica acerca del prejuicio e intenta comprender mejor tanto su naturaleza como aquellos mecanismos que contribuyen a su perpetuación, y repasa detenidamente las distintas estrategias que han sido propuestas con la intención de combatirlo y reducir el impacto de sus nocivas consecuencias. La pertinencia y el alcance de este trabajo quedarían justificados por las enormes posibilidades que la reducción del prejuicio podría brindar a la consecución de sociedades más cohesionadas.

\section{La dificultad de combatir el prejuicio: algunos elementos para el análisis}

Aunque han sido muchas las distintas definiciones que se han propuesto, fue Allport quien aportó una de las primeras y más relevantes definiciones del prejuicio. En su libro The nature of prejudice, presenta el prejuicio como «una antipatía basada sobre una defectuosa e inflexible generalización. Puede ser sentida o expresada. Puede ser dirigida hacia un grupo en su conjunto o hacia un individuo en tanto que es un miembro de ese grupo" (Allport, 1954: 10).

En general, desde Adorno (Adorno et al., 1950), un gran número de autores han coincidido en señalar que el prejuicio constituye un elemento negativo, irracional, erróneo y rígido (Harding et al., 1969). Negativo por las nocivas 
consecuencias sociales que el prejuicio puede provocar. Irracional porque parece estar más ligado a aspectos emocionales que racionales. Erróneo porque el prejuicio es percibido como el resultado de distorsiones y limitaciones de nuestro conocimiento. Y, finalmente, rígido porque en muchos casos parece perpetuarse incluso en aquellas ocasiones en que la realidad parece desmentirlo.

En todo caso, y a pesar de las negativas consecuencias que implica el hecho de emitir un juicio o una evaluación sin disponer de la totalidad de la información necesaria para ello, la generación de prejuicios es muy frecuente en las relaciones sociales y, como veremos a continuación, extraordinariamente difícil de combatir (Monteith et al., 1994).

\section{El prejuicio como la profecía que se cumple a si misma}

Modificar un prejuicio es una tarea realmente complicada por varias razones. En primer lugar, porque probar que el prejuicio es injusto, falso o incorrecto es difícil, no sólo debido a que la realidad social no se deja medir de un modo objetivo e inmediato, sino porque se trata de una realidad que se encuentra en permanente cambio y construcción. Nuestras percepciones, definiciones y expectativas acerca del mundo social tienen un importantísimo papel a la hora de configurar esa misma realidad social que estamos conociendo. De este modo, el prejuicio no es simplemente una respuesta evaluativa respecto a un colectivo, sino que, además, tiene la capacidad de influir sobre ese mismo colectivo que lo sufre.

Como Jones (1990) puso de manifiesto, las expectativas de las personas pueden influir, aun de modo inconsciente, sobre cualquier interacción interpersonal, de manera que acaben provocando su propia confirmación. Este fenómeno, denominado por Merton como «la profecía que se cumple a sí misma» (self-fulfilling prophecy; Merton, 1948), parte de la formulación que realizó Thomas al afirmar que «siempre que los hombres definen las situaciones como reales son reales en sus consecuencias». Esto es, la definición que se hace de una situación concreta pasa a formar parte de la situación definida, lo cual afecta a su desarrollo posterior.

En el ámbito de las relaciones interpersonales, este mismo fenómeno recibe el nombre de confirmación conductual (Behavioral confirmation effect; Snyder, 1992; Jones, 1990). Las expectativas que se tienen respecto a otro individuo influyen en el comportamiento de dicho individuo, lo cual favorece que se cumplan tales expectativas.

Como puede suponerse, el prejuicio, y más concretamente los estereotipos (su elemento cognitivo), son intentos de definir la realidad social y, por tanto, actúan como fuente de expectativas. Es decir, el prejuicio y los estereotipos nos impulsan a esperar un comportamiento o un modo de ser determinado respecto a un colectivo social. La mayoría de las investigaciones que se centran en el estudio de los procesos implicados en la confirmación conductual asumen que el perceptor desempeña un papel dinámico y fundamentalmente activo a la hora de provocar la evidencia confirmatoria (Neuberg, 1994; Snyder, 1992). 
Por otro lado, si adoptamos la perspectiva, no del que sostiene el estereotipo, sino del colectivo o individuo que lo sufre, existe a su vez otro proceso (íntimamente relacionado con el anterior) que ayuda a explicar por qué las creencias que mantenemos sobre las personas o los grupos son capaces de llegar a formar parte de la realidad aun pudiendo ser inicialmente falsas. Y es que los individuos tienden a adecuarse a la idea que se tiene de ellos y son proclives a comportarse, por tanto, en función de lo que de ellos se espera. Este peculiar mecanismo ha sido denominado «efecto Pigmalión» por Rosenthal y Jacobson (1968), quienes demostraron que las expectativas que los profesores poseen acerca de los alumnos tienen un papel absolutamente fundamental en el desarrollo de su rendimiento intelectual. Cuanto mejores creían que eran sus alumnos, mejores llegaban a ser dichos alumnos.

Como sostiene el interaccionismo simbólico (Blumer, 1969), la identidad de los individuos (the self) se constituye de un modo interactivo e interaccional. Es decir, la definición que hacemos de nosotros mismos se deriva, en parte, de las definiciones que los demás hacen de nosotros, y viceversa. A su vez, la teoría de la identidad social (Tajfel y Turner, 1986) defiende que las personas no actúan como meros individuos, sino como seres sociales que extraen una parte importante de su identidad de los grupos humanos y de las categorías sociales a las que pertenecen. Por tanto, los miembros de grupos sociales estereotipados que son objeto de prejuicio pueden acabar interiorizando y haciendo suya la definición negativa que se hace de ellos, con lo cual pasa a formar parte de su propia identidad y se refleja en su conducta (Bonnot y Croizet, 2007).

Así pues, en la medida en que los estereotipos y el prejuicio establecen expectativas, cumplen una función prescriptiva y, consiguientemente, definen no sólo identidades, sino también roles (Rudman y Glick, 2001). Por este motivo, en la mayoría de los casos, los distintos colectivos tienden a amoldarse a los roles que se consideran apropiados y congruentes con sus características y sus estereotipos supuestos.

Por otro lado, aun cuando el individuo estereotipado puede no haber interiorizado, asimilado y hecho suyo el contenido del estereotipo, sigue siendo posible que el mero conocimiento y la subsiguiente activación automática del estereotipo tenga consecuencias negativas sobre su conducta (para una revisión de dicho concepto, véase Wheeler et al., 2001).

Puede concluirse, por tanto, que son varios los posibles procesos y mecanismos mediante los cuales un estereotipo, que incluso podría ser inicialmente falso o equivocado, puede incidir de tal manera en lo real, que llegue a convertirse en verdadero. El prejuicio y el estereotipo impregnan la realidad de tal manera que pueden transformarla adaptándola a ellos mismos, lo cual provoca que sea aún más difícil la tarea de combatirlos.

\section{Mecanismos que hacen resistente al prejuicio}

Igualmente, reducir el prejuicio y cambiar los estereotipos es una labor compleja y difícil, ya que son varios los mecanismos mediante los cuales los estereo- 
tipos y el prejuicio tienden a perpetuarse. El prejuicio, al igual que las demás actitudes, guían la exposición, la atención y la codificación de la información a través de la percepción y el juicio, así como su recuperación de la memoria siempre en una dirección congruente con la propia actitud (Bodenhausen et al., 1999). Es decir, las personas tendemos a exponernos, a seleccionar y a recordar con mayor facilidad aquella información que es coherente con nuestras propias creencias y actitudes. El prejuicio, por tanto, como cualquier otra actitud, guía la búsqueda de información que refuerce o valide dicha actitud e ignora selectivamente la información que no coincide con la misma.

Por otro lado, Sherman y colaboradores (2005) encontraron que cuando los individuos más prejuiciosos evalúan a una persona que pertenece a un grupo estereotipado (e. g., inmigrante), tienden a fijarse más detenidamente (ya que les sorprende) en aquella información que no encaja con el estereotipo que tienen en mente, pero, sin embargo, a pesar de prestar más atención a esa información, no la usan para elaborar sus juicios sobre la persona en cuestión. Una vez que un individuo ha desarrollado y ha almacenado una idea resistente acerca de los rasgos característicos de una persona o de un grupo, tiende a basar sus juicios sociales en esa idea previa en vez de usar la información que se encuentre en episodios posteriores, por mucho que llame su atención.

Una de las explicaciones de este fenómeno es que los individuos con un alto grado de prejuicio tienden a desestimar los comportamientos no estereotípicos, atribuyéndolos a motivos externos y explicaciones circunstanciales, mientras que los comportamientos consistentes con el estereotipo los atribuyen a motivos internos y a rasgos característicos del sujeto (Pettigrew, 1979). Estos resultados concuerdan, además, con la tesis de que la gente acepta fácilmente y con escaso escrutinio aquella información que concuerda con sus deseos y sus expectativas y, sin embargo, es mucho más exigente cuando se trata de aceptar información no deseada o esperada y, en consecuencia, la examina con más detenimiento con intención de rebatirla (Trope y Liberman, 1996).

Entre las estrategias con las que contamos para conservar inalterados nuestros prejuicios y estereotipos, podemos resaltar la racionalización, la excepcionalización y la subcategorización. Un ejemplo característico de la primera de ellas es el citado error último de atribución, mediante el cual descartamos la validez de un comportamiento positivo y no estereotípico explicándolo, no como una consecuencia de rasgos internos del sujeto (habilidad y competencia, por ejemplo), sino como resultado de factores y causas externas (circunstancias especiales, suerte, ayuda exterior, etc.) (Pettigrew, 1979). A su vez, mediante la segunda de las estrategias posibles, podemos invalidar la información que contradice nuestros prejuicios (e. g., conocer a uno o varios individuos de un grupo social cuyo comportamiento o modo de ser no se corresponde con el estereotipo que nos hemos formado de dicho grupo), puesto que la consideramos un caso excepcional que prueba la regla general (Yzerbyt et al., 1999). Por último, la tercera estrategia, en una línea similar a la anterior, consiste en desarrollar subcategorías en las que introducir los casos que no encajan con el estereotipo que se sostiene sobre un grupo, lo cual da a entender que tales 
casos no pueden ser considerados verdaderos miembros del grupo (Kunda y Oleson, 1995).

\section{El respaldo social del prejuicio como garantía de permanencia}

Otro de los motivos que dificultan aún más la ya de por sí complicada tarea de combatir el prejuicio es el hecho de que se trata de un fenómeno de naturaleza grupal. Es decir, una de sus características principales es la de ser compartido y transmitido socialmente (Sinclair et al., 2005). Esto es de especial importancia, puesto que, como señaló Festinger en su propuesta teórica sobre la validación de la realidad social (1950), cualquier individuo es influido por sus semejantes (e. g., miembros de un mismo grupo o aquellos con los que tiene algún rasgo en común), al formar juicios sobre realidades poco objetivas, ya que adquiere una mayor confianza en la corrección de sus juicios según sus semejantes estén de acuerdo con él. Además, cuanto más unido se encuentra el grupo, mayor influencia ejerce sobre las actitudes que sus miembros tienen respecto a estímulos ambiguos (Festinger et al., 1951). Alternativamente, Deutsch y Gerard (1955) pusieron de manifiesto el peso del grupo sobre nuestras propias convicciones al replicar y extender los hallazgos de los conocidos experimentos de Asch (1958), en los que los participantes acababan asumiendo juicios evidentemente erróneos sólo para no discrepar con la opinión mayoritaria.

Así pues, el apoyo social que sustenta al prejuicio y a los estereotipos, y que actúa además como un argumento más a favor de su uso y su existencia, los protege ante cualquier posible intento de desmentirlos. Como la investigación pasada ha puesto de manifiesto, no solamente concedemos un gran valor a cualquier creencia o actitud defendida por una amplia mayoría, sino que también nos es francamente difícil contradecir las opiniones grupales, aun a sabiendas de que no se corresponden con la realidad.

\section{La importancia del prejuicio en la formación de la identidad personal}

Por último, es importante señalar que el prejuicio, quizá con más probabilidad que cualquier otra actitud, puede cumplir una gran cantidad de funciones emocionales, como, por ejemplo, la definición de la propia identidad y, en consecuencia, el ensalzamiento de la propia autoestima.

Según la teoría de la autocategorización (Turner, 1987), las personas no actúan como meros individuos, sino como seres sociales que extraen una parte importante de su identidad de los grupos humanos y de las categorías sociales a las que pertenecen. De hecho, la teoría de la identidad social no sólo esclarece nuestra preferencia natural por el propio grupo, sino que también explica por qué podemos llegar a despreciar y criticar a individuos pertenecientes a otros grupos. Como las personas evalúan sus grupos mediante procesos de comparación intergrupal, buscan pertenecer a colectivos que son evaluados de manera más positiva que los demás. De este modo, la necesidad de mantener una visión positiva acerca del propio grupo provoca que una de las posibles 
estrategias para lograrlo sea menospreciar y denigrar a otros grupos, así como limitar sus oportunidades (Tajfel y Turner, 1986).

Cuando ese es el caso, el prejuicio se convierte automáticamente en una actitud importante para las personas que lo sostienen, y las actitudes importantes, según han señalado Zuwerink y Devine (1996), son más resistentes frente a posibles mensajes, informaciones o ataques contraactitudinales. Uno de los motivos que hacen que las actitudes importantes sean especialmente resistentes es que cuanto más importante sea para la persona la actitud en sí misma (no el objeto de actitud), mayor será la probabilidad de que tales individuos realicen una búsqueda y un procesamiento sesgado de la información relacionada con el objeto de actitud (Briñol et al., 2007).

En resumen, la capacidad del prejuicio para incidir sobre la realidad social y transformarla a su imagen y semejanza, el relevante consenso social que le confiere su naturaleza grupal, los distintos mecanismos psicológicos que permiten y facilitan su perpetuación y la importancia que, en la mayoría de los casos, representa para aquellos que lo sostienen constituyen un conjunto de obstáculos que hacen del prejuicio una actitud especialmente resistente y difícil de cambiar.

\section{Estrategias de reducción de prejuicio: soluciones a un problema social}

Desde que, a mediados del siglo Xx, el concepto de prejuicio fue adoptado mayoritariamente para expresar lo que se consideraban actitudes profundamente injustas e irracionalmente negativas hacia grupos diferentes, han sido muchos los intentos por combatir las causas y los efectos sociales de tan extendido fenómeno. A pesar de su complejidad, se presenta seguidamente una breve clasificación de todos ellos estableciendo tres niveles generales de análisis: social, interpersonal e individual. Se trata, con ello, de aportar posibles soluciones encaminadas a erradicar o, cuanto menos, minimizar este importante problema social que afecta a los distintos tipos de exogrupos presentes en una sociedad ${ }^{2}$.

\section{Nivel social: la importancia de las políticas públicas, la educación $y$ los medios de comunicación en la reducción del prejuicio}

La estructura social posee una influencia innegable sobre el prejuicio, puesto que afecta al conjunto de relaciones sociales e interpersonales que tienen lugar en su interior. En este sentido, Russell (1961) mostró cómo las normas sociales que favorecen la discriminación son capaces de contaminar las posibles interacciones que tienen lugar entre los distintos grupos sociales (véase también

2. Como se había planteado en la introducción de este trabajo, los mecanismos por los que se forman y perduran los prejuicios son comunes al conjunto de los exogrupos y minorías. Por tanto, las estrategias de reducción del prejuicio que presentamos podrían ser también extensibles a todos esos grupos minoritarios, independientemente del motivo de su constitución (migraciones, religión, género, etc.). 
Rodríguez, Betancor y Delgado, 2009). Además, y como es bien sabido, las sociedades democráticas albergan niveles menores de conflicto y de discriminación y, paralelamente, mayores niveles de tolerancia política y social que las sociedades autoritarias (Sullivan y Transue, 1999).

Por ello, la gran mayoría de los investigadores sociales han percibido que para intentar cambiar las actitudes de las personas era necesario, en primer lugar, cambiar el contexto en el que éstas se encontraban inmersas (Sherif y Sherif, 1953). De este modo, una de las primeras y más básicas estrategias para reducir el prejuicio consistió en el empleo de políticas públicas. Las diferentes políticas públicas pueden restaurar derechos y privilegios a los grupos que han sido previamente excluidos $y$, en consecuencia, incrementar sus recursos y sus oportunidades. No obstante, es importante que la implantación de dichas políticas públicas sea vista como algo justo y necesario, ya que, en caso contrario, podrían incluso reforzar el prejuicio respecto a aquellas minorías que intenta proteger, con lo cual extendería la idea de que son un grupo realmente inferior (ya que requiere una asistencia especial) y que va a perjudicar al resto de la sociedad consumiendo sus recursos. En concreto, Maio y Esses (1998) llevaron a cabo un estudio en el que constataron experimentalmente que cuando se informaba que un nuevo grupo de inmigrantes iba a disponer de ayudas y a beneficiarse de posibles políticas de discriminación positiva, éstos eran evaluados de un modo más negativo e incluso la inmigración en general era considerada de un modo menos favorable.

Por otro lado, la eficacia de las políticas públicas radica también en que, como señala Aronson (1999), cambiar los comportamientos de las personas a través de mandatos legales es el primer paso para cambiar sus actitudes y sus pensamientos de un modo eficaz, ya que la tendencia existente en las sociedades democráticas a identificar lo legal con lo moral y lo lícito otorga a la ley un valor educativo y legitimador extraordinario. Como indican diferentes investigaciones acerca de la disonancia cognitiva y el cambio de actitudes, la conformidad conductual con leyes y normas antidiscriminatorias desemboca en unas actitudes más favorables hacia las minorías en cuestión (Leippe y Eisenstadt, 1994). La teoría de la disonancia cognitiva establece que cuando las personas experimentan inconsistencia entre su manera de pensar y la de actuar, sienten un malestar y una incomodidad que les llevará a un cambio conductual o cognitivo con la finalidad de reducir dicha inconsistencia (Festinger, 1957). Así, cuando los individuos son impelidos a actuar de un modo que contradice su actitud (e. g., a favor de un grupo minoritario) y, por tanto, inconsistente con su manera de pensar, experimentan un malestar que pueden resolver modificando su actitud en la dirección del comportamiento realizado (para una revisión, véase Cooper y Fazio, 1984).

Otro de los principales intentos de mejorar las actitudes de las personas hacia los grupos que son objeto de prejuicio ha sido a través de la utilización de comunicaciones persuasivas. Gran parte de estos intentos de reducir el prejuicio a través de mensajes, informaciones y argumentos se basa en el supuesto de que uno de los principales factores que sustentan y promueven el prejuicio es 
la ignorancia (Stephan y Stephan, 1984). Ese es el motivo, por tanto, de que, en el ámbito escolar y educacional, se hayan realizado innumerables intervenciones destinadas a aumentar el conocimiento y la valoración de otros grupos sociales religiosos o étnicos (Bigler, 1999), así como a subrayar las múltiples y positivas contribuciones que esos mismos grupos han realizado (Lynch, 1986). Del mismo modo, un número importante de intervenciones han incluido directamente educación explícita sobre el prejuicio, los estereotipos y la discriminación y se han centrado en argumentar y favorecer la comprensión de sus fundamentos irracionales, así como de sus raíces históricas, económicas y estructurales (McGregor, 1993).

A su vez, fuera de las aulas y a un nivel más general, otra estrategia de carácter global que ha mostrado una gran capacidad para influir en la opinión pública de cualquier sociedad es aquélla que se basa en el uso de los medios de comunicación de masas. Desde los primeros estudios acerca de los posibles efectos de la propaganda (Hovland et al., 1949), quedó clara la capacidad que los mensajes colectivos poseían para moldear las opiniones y las actitudes grupales de los miembros de la sociedad en ambas direcciones, tanto para fomentar el prejuicio (Lasswell, 1928), como para reducirlo (Flowerman, 1949). Entre los distintos empeños para reducir el prejuicio a través de los medios de comunicación, podemos encontrar campañas educativas (entertainment-education; Singhal et al., 2004), campañas publicitarias en televisión (Vrij et al., 1996), en radio (Paluck, 2009) y en vallas (Vrij y Smith, 1999). A su vez, las series de ficción emitidas a través de la televisión o de la radio (Ball-Rokeach et al., 1981) pueden ser empleadas para intentar establecer de un modo explícito normas sociales de igualdad y tolerancia o para expresar dichas normas de un modo que los espectadores puedan inferirlas con facilidad del comportamiento de los personajes, del mismo modo que las infieren de los demás miembros de la sociedad.

Anteriormente apuntamos que, debido al peso que el consenso ejerce sobre nuestras propias opiniones (Asch, 1958), una de las grandes dificultades para combatir el prejuicio era el apoyo generalizado con el que cuenta por parte de la comunidad. Así, diversos estudios han revelado la posibilidad de reducir el prejuicio e incluso el uso y la activación automática de los estereotipos proporcionando información sobre su bajo consenso social (Stangor et al., 2001). Por este mismo motivo, los investigadores de los medios de comunicación señalan que su gran eficacia a la hora de establecer y generalizar lo que la mayoría de la gente piensa o hace es una herramienta de una enorme utilidad para reducir el apoyo social de cualquier postura indeseable, incluida el prejuicio (Bandura, 2004).

\section{Nivel interpersonal: propuestas a partir de la hipótesis del contacto}

De entre todas las estrategias que podrían encuadrarse en este apartado, la hipótesis del contacto intergrupal es, sin ninguna duda, la que más atención ha recibido por parte de los investigadores sociales (Pettigrew y Tropp, 2006; Pet- 
tigrew, 1998; Allport, 1954; Watson, 1947; Williams, 1947; para una revisión en castellano, véase Gómez, 2004). Esta hipótesis propone, principalmente, que el contacto entre grupos puede lograr una mejora en las relaciones intergrupales y reducir el prejuicio. Ahora bien, para que el contacto entre diferentes grupos sea eficaz y mejore las relaciones entre ellos, es necesaria la presencia de ciertos requisitos fundamentales.

A. Evidencia empirica. Ya en los años treinta era posible encontrar la idea de que el contacto intergrupal podría constituir un posible modo de reducir el prejuicio. Al estudiar la relación entre distintos factores y las actitudes hacia 39 grupos sociales diferentes, Zelings y Hendrickson (1933) encontraron que el factor más relacionado con la tolerancia social era el grado en que los individuos afirmaban mantener una relación de proximidad y compañerismo (acquaintanceship) con los distintos grupos.

Sin embargo, pronto también comenzaron a surgir las voces que afirmaban que no cualquier tipo de contacto era igualmente válido, sino que solamente aquellas situaciones en las que se despertaban sentimientos positivos (Watson, 1947), que poseían una estructura adecuada de colaboración entre iguales (Williams, 1947) y que tenían lugar en un contexto en el que se compartían objetivos comunes que requerían una total cooperación (Sherif et al., 1961) conducían a una verdadera mejora de las relaciones entre diferentes grupos.

B. Condiciones: ¿En qué situaciones funciona? Éste era el contexto teórico y empírico en el que Allport (1954) formuló su conocida hipótesis del contacto intergrupal. Su versión sostenía que el contacto intergrupal produce un efecto positivo sólo en aquellas situaciones en las que concurrían cuatro rasgos necesarios: (1) un estatus equivalente en la situación en la que se produce el contacto; (2) cooperación intergrupal; (3) objetivos comunes, y (4) apoyo y respaldo por parte de las autoridades, la ley o las costumbres sociales.

Generalmente, la investigación posterior ha respaldado los cuatro prerrequisitos establecidos por Allport. En lo referente a compartir un mismo estatus, Jackman y Crane (1986) pusieron de manifiesto los efectos negativos que se producían cuando el contacto tenía lugar con miembros de otros grupos con un estatus inferior.

El respaldo empírico es especialmente significativo en el caso de la cooperación intergrupal (Bettencourt et al., 1992). La reconocida eficacia de este principio ha dado lugar a técnicas e intervenciones tan conocidas como el aprendizaje cooperativo (Johnson y Johnson, 2000) y la clase rompecabezas (The jigsaw classroom; Aronson y Patnoe, 1997).

Del mismo modo, compartir unas metas u objetivos comunes supone un requisito más para el éxito de la interacción entre grupos. No obstante, como han señalado Gaertner y colaboradores (1999), este factor podría ser menos importante de lo que Allport (1954) sugirió originalmente cuando no va unido a una interacción de tipo cooperativo.

La evidencia empírica disponible también muestra que el contacto intergrupal es realmente eficaz cuando tiene lugar en un contexto de apoyo legal y social. Cuando, por el contrario, el contacto tiene lugar en contextos donde 
esta condición se encuentra ausente, los resultados son, en muchos casos, contraproducentes (Russell, 1961).

Por último, además de las condiciones señaladas hasta el momento, las investigaciones más recientes coinciden en subrayar la oportunidad de amistad (aquaintance potencial) como otro de los factores determinantes para que el contacto intergrupal desemboque en la mejora de las relaciones entre diferentes grupos sociales (Molina y Wittig, 2006; Pettigrew, 1998).

C. Procesos implicados: ¿Por qué funciona? Según fue consolidándose el consenso en torno a los requisitos necesarios para un eficaz contacto intergrupal, el interés y la atención pasó de concentrarse en cuáles eran esas condiciones a por qué esas condiciones eran importantes.

En primer lugar, es interesante recordar que una de las principales causas de la existencia del prejuicio hunde sus raíces en un fenómeno psicosocial denominado sesgo endogrupal, es decir, en la natural preferencia que todos sentimos hacia los miembros de nuestro propio grupo. Por eso, una situación de tipo cooperativo enfocada al logro de un objetivo común en un contexto de igualdad y de posible amistad constituye un ámbito propicio para dejar de concebir a un individuo de otro grupo simplemente como miembro indiferenciado de una categoría y pasar a considerarlo como un individuo singular. Este proceso de diferenciación ha recibido, comúnmente, el nombre de decategorización (Bettencourt et al., 1992). No obstante, Hewstone y Brown (1986) pusieron de manifiesto el peligro de la subcategorización. Es decir, sugirieron que cuando tenía lugar un contacto positivo entre miembros de diferentes grupos bajo condiciones de una marcada personalización, existía el peligro de situarlos dentro de una subcategoría alternativa y, por tanto, de que la evaluación positiva que se pudiera producir hacia el individuo concreto no se generalizara al conjunto del grupo.

A su vez, dicha situación cooperativa constituye un ámbito propicio no sólo para que, como hemos visto, tenga lugar un proceso de decategorización, sino que es también el contexto oportuno para que se produzca un proceso de recategorización. Según el enfoque de la recategorización contenido en el modelo de identidad común intragrupal (Common In-group Identity Model; Gaertner y Dovidio, 2000; para una revisión en castellano, véase Gómez, 2003), el prejuicio puede ser reducido de manera más eficaz cuando individuos de grupos diferentes pasan a formar parte de un mismo grupo de orden superior, con lo cual quedan englobados por una nueva identidad común. Así pues, no se trata solamente de hacer menos visible la distinción grupal o categorial (decategorización), sino que se crea una nueva identidad compartida (recategorización) que puede sustituir y reemplazar a la anterior (o coexistir con ella).

El contacto intergrupal facilita, a su vez, la adquisición de nueva información acerca de los miembros de otros grupos, lo cual es especialmente útil en aquellos casos en los que el prejuicio surge como efecto de la ignorancia (Stephan y Stephan, 1984). Igualmente, este mayor conocimiento puede ayudar a contemplar y considerar a los demás de un modo individualizado y personalizado, lo cual favorece el ya mencionado proceso de decategorización. 
Por último, es importante apuntar que todos los procesos implicados en la reducción del prejuicio a través del contacto intergrupal señalados hasta aquí poseen una cualidad común: todos ellos facilitan la reducción de la ansiedad intergrupal, que constituye uno de los principales obstáculos para cualquier relación intergrupal (Stephan et al., 2002).

\section{Nivel individual: la necesidad de controlar los factores psicológicos}

Si bien los factores psicológicos quedan ciertamente condicionados por los niveles social e interpersonal vistos con anterioridad, no cabe duda de que ciertos elementos individuales adquieren gran protagonismo en las estrategias de reducción del prejuicio. En este sentido, uno de los elementos considerados tradicionalmente como facilitadores del prejuicio ha sido el pensamiento simple y falto de matices. Por tal motivo, diferentes enfoques han argumentado que técnicas como el entrenamiento en un pensamiento más complejo y variado (Complexity training; Gardiner, 1972) y el entrenamiento en razonamiento estadístico (Schaller et al., 1996) pueden ayudar a reducir la elaboración de toscas generalizaciones acerca de los demás grupos sociales.

Por otro lado, diferentes investigadores afirman que otro modo eficaz de mejorar las relaciones intergrupales puede lograrse reduciendo la distancia psicológica entre los individuos con prejuicios y los individuos victimas del prejuicio a través de la empatía (Stephan y Finlay, 1999). Tal y como indica la investigación al respecto, la ausencia de empatía está asociada con conductas y actitudes antisociales, mientras que las estrategias destinadas a desarrollar la empatía conllevan un elevado número de consecuencias positivas sobre las actitudes y el comportamiento.

Asimismo, la ya mencionada teoría de la disonancia cognitiva (Festinger, 1957) ha sido empleada en diversas estrategias destinadas a reducir el prejuicio de las personas mediante el uso de ejercicios como, por ejemplo, la confrontación interpersonal (Czopp et al., 2006), que hacen explícita la inconsistencia entre algunas de sus creencias y valores (e. g., igualdad, justicia, etc.) con sus conductas discriminatorias. Así, el reconocimiento de discrepancias entre el comportamiento personal (i. e., mostrar tendencias prejuiciosas) y el autoconcepto (i. e., considerarse una persona justa e igualitaria) genera sentimientos negativos hacia uno mismo que pueden promover un comportamiento más igualitario y tolerante en situaciones sucesivas.

Por otra parte, las investigaciones en las que se pide a los participantes que expongan y defiendan públicamente posiciones contrarias a las propias, con el objetivo de evaluar dicha exposición sobre las propias convicciones, mostraron que el role-playing (Janis y King, 1954) puede constituir una estrategia eficaz de cara a mejorar las evaluaciones hacia grupos minoritarios (McGregor, 1993).

Por otro lado, como el prejuicio y los estereotipos están constituidos por asociaciones afectivas y semánticas que son aprendidas socialmente y que tienen unas raíces profundamente arraigadas en nuestra estructura psicológica, es posible que, aun sin compartirlos, se activen de un modo automático y 
actúen en nosotros sin nuestro conocimiento y, lo que es peor, sin nuestro consentimiento (Devine, 1989). Este carácter automático ha sido una de las principales causas de que diversos investigadores hayan defendido que la presencia y el influjo de los estereotipos y los prejuicios en nuestra vida es prácticamente inevitable (Bargh, 1999). No obstante, cada vez son más numerosos los estudios que revelan la naturaleza moldeable de los estereotipos y los prejuicios automáticos (para una revisión, véase Blair, 2002). Los primeros intentos de suprimir la activación automática de los estereotipos mostraron que los esfuerzos conscientes de negar los estereotipos tenían el irónico resultado de alimentar aún más el prejuicio, ya que aumentaban su accesibilidad (Macrae et al., 1994). Sin embargo, otros estudios indican que las estrategias de supresión pueden ser eficaces cuando van acompañadas por intenciones concretas de implementación (para una revisión, véase Monteith et al., 1998). Kawakami y colaboradores (2005), a su vez, encontraron que se podía reducir la activación automática del prejuicio y de los estereotipos mediante la negación continuada y reiterada de las asociaciones estereotípicas.

Por último, otros intentos de alterar las asociaciones afectivas y cognoscitivas que componen el prejuicio han estado basados, no en la supresión de los estereotipos, sino en la generación de imágenes y asociaciones contraestereotípicas (Blair, 2002). En este mismo sentido, Dasgupta y Greenwald (2001) mostraron que se podía reducir el prejuicio automático mediante la exposición a miembros no estereotípicos de grupos estigmatizados. En concreto, los participantes que estuvieron expuestos a fotografías de americanos famosos, admirados y de raza negra (e. g., Martin Luther King) y a fotografías de americanos despreciables de raza blanca (e. g., un asesino en serie como Jeffrey Dahmer) mostraron un nivel de prejuicio automático significativamente menor que el de los individuos asignados al grupo control. En una línea relativamente similar y también como alternativa a la ineficaz estrategia de supresión, Sassenberg y Moskowitz (2005) propusieron que la activación automática de los estereotipos puede ser superada simplemente induciendo a las personas a pensar de un modo diferente y creativo.

\section{Conclusiones}

El proceso de globalización en el que está inmerso el planeta desde hace más de treinta años se caracteriza tanto por los aspectos económicos - con la generalización del capitalismo-, políticos — con la extensión de la democracia—y culturales — con la difusión de la cultura occidental—, como por la circulación planetaria de un flujo constante de información, bienes y personas. En relación con este último aspecto, nos resulta cada vez más familiar tanto la presencia de minorías de religión, etnia o cultura diferentes, así como los diferentes debates en torno a las políticas con las que se deben gestionar estos nuevos contextos pluriculturales (Innerarity y Acha, 2010).

De este modo, la velocidad, la profundidad y la generalización de estos movimientos migratorios implica la aparición de un número creciente de socie- 
dades - como la española — que han pasado en un corto espacio de tiempo de la homogeneidad a la pluralidad cultural. Indudablemente, este nuevo panorama hace necesaria una mínima convivencia entre grupos de composición diferente, pero, como se ha resaltado en este trabajo, es frecuente que la naturaleza multicultural de las nuevas sociedades de hoy en día se convierta en un caldo de cultivo especialmente propicio para que surjan valoraciones y opiniones negativas y discriminatorias (i. e., prejuicios) hacia los grupos minoritarios.

Por otro lado, si bien la dirección, la intensidad y el nivel del prejuicio entre dos grupos cualesquiera vienen determinados, en parte, por las características de las relaciones entre esos grupos (competencia sobre recursos escasos, dominación de un grupo sobre otro, etc.), es precisamente su gran persistencia y la notable dificultad para modificarlos una vez que han sido asumidos por los individuos lo que complica en gran medida la gestión de los prejuicios en las sociedades actuales.

Como se ha puesto de manifiesto en este trabajo, existen diversos mecanismos que hacen del prejuicio una actitud especialmente difícil de cambiar. En primer lugar, porque constituye un elemento con el que construimos la realidad $y$, aunque se base en generalizaciones o simplificaciones, finalmente es adoptado e interiorizado tanto por los individuos que lo sostienen como por los que lo sufren. En segundo lugar, porque es habitual que, una vez que se ha interiorizado un prejuicio, éste se asuma como verdadero y se refute toda nueva información contraestereotípica como estrategia para mantener la consistencia con nuestra forma de pensar. En tercer lugar, debido a que el prejuicio no lo construimos de forma individual, sino que se apoya en el respaldo del grupo que lo sostiene, lo que le da validez social y lo hace más resistente. Y, por último, porque los prejuicios, como otras actitudes, cumplen una función identitaria muy importante que nos asimila a las características del grupo de pertenencia (endogrupo) y, paralelamente, nos diferencia del grupo de comparación (exogrupo), lo que dificulta que modifiquemos esa actitud, porque ello implicaría perder en parte nuestra identidad grupal.

En todo caso, a pesar de la gran cantidad de dificultades que encontramos a la hora de reducir el prejuicio, los investigadores sociales han logrado desarrollar un número considerable de estrategias que han mostrado ser eficaces para mejorar las actitudes intergrupales. Con fines analíticos, dichas estrategias han sido organizadas aquí bajo tres niveles de estudio: el social, el interpersonal y el individual.

Desde el punto de vista social, se ha destacado que lo más importante es cambiar el contexto en el que se generan y se difunden las actitudes prejuiciosas. En este sentido, los diferentes estudios referenciados ponen de manifiesto la importancia y la necesidad de elaborar normativas públicas, diseñar políticas educativas y plantear campañas comunicativas que incidan en la creación de un espacio social de mayor tolerancia cultural y libre de prejuicios hacia las minorías. Desde el plano interpersonal, y aun cuando la convivencia entre diferentes grupos puede despertar en un primer momento ciertas actitudes discriminatorias, la literatura aquí citada ha mostrado que, si se cumplen ciertos 
requisitos fundamentales (cooperación, búsqueda de objetivos comunes, respaldo legal y social, etc.), el impulso del contacto intergrupal reduce la evaluación negativa de unos grupos hacia otros. Por último, desde el plano individual, se aboga por acciones que traten de incidir en los factores psicológicos a partir del aumento de la empatía, eliminando la disonancia cognitiva o suprimiendo la activación automática de los estereotipos en base a la generación de imágenes y asociaciones contraestereotípicas.

Es indudable que la solución de los problemas sociales obedece, como también su explicación, a múltiples factores. Pero entendemos que los resultados de todas estas investigaciones permiten afrontar con un mayor optimismo la batalla por reducir la discriminación social a la que se ven sometidas las minorías en general y los inmigrantes en particular. Consideramos, en definitiva, que, en la medida en que las sociedades sean capaces, desde las diferentes instancias públicas, medios de comunicación, responsables educativos y sociedad en general, de hacer efectivas las estrategias para la reducción del prejuicio apuntadas en estas páginas, la convivencia entre los diferentes grupos tenderá a mejorar, con lo cual se alcanzará una armonía absolutamente necesaria para el buen funcionamiento de las nuevas sociedades pluriculturales.

\section{Referencias bibliográficas}

Adorno, T.; Frenkel-Brunswick, E.; Levinson, D. y SAnford, N. (1950). The authoritarian personality. Nueva York: Harper.

Allport, G. W. (1954). The nature of prejudice. Cambridge: Addison-Wesley.

Alonso, J. A. (2011). «Migración internacional y desarrollo: una revisión a la luz de la crisis». CDP Background Paper, 11, 1-66.

ÁlvareZ GÁlveZ, J. (2009). «La representación mediática de la inmigración. Entre el encuadre y el estigma». Revista del Ministerio de Trabajo e Inmigración: Migraciones Internacionales, 80, 61-80.

ARONSON, E. (1999). The social animal. Nueva York: Freeman.

Aronson, E. y Patnoe, S. (1997). The Jigsaw classroom. Nueva York: Longman.

AsCH, S. (1958). «Effects of group pressure upon the modification and distortion of judgments». En: MACOBY, E. E. et al. Readings in Social Psychology edited. Nueva York: Holt, Rinehart, and Winston.

Ball-RokeACH, S. J.; Grube, J. W. y RoKeACH, M. (1981). «Roots: The next generation. Who watched and with what effect?». Public Opinion Quarterly, 45 (1), 58-68.

BANDURA, A. (2004). «Social cognitive theory for social and personal change enabled by the media». En: Singhal, A.; Cody, M.; Rogers, E. y Sabido, M. (eds.). Entertainment-education and social change. Mahwah, NJ: Lawrence Erlbaum.

BARGH, J. A. (1999). "The cognitive monster: The case against the controllability of automatic stereotype effects». En: CHAIKEN, S. y TrOPE, Y. (eds.). Dual process theories in social psychology. Nueva York: Guilford.

Bettencourt, B. A.; Brewer, M. B.; Rogers-Croak, M. R. y Miller, N. (1992). «Cooperation and the reduction of intergroup bias: The roles of reward structure and social orientation». Journal of Experimental Social Psychology, 28, 301-319.

BigLER, R. S. (1999). «The use of multicultural curricula and materials to counter racism in children». Journal of Social Issues, 55, 687-706. 
BlaIR, I. V. (2002). "The malleability of automatic stereotypes and prejudice». Personality and Social Psychology Review, 6, 242-261.

Blumer, H. (1969). Symbolic interaction: Perspective and method. Englewood Cliffs, NJ: Prentice-Hall.

Bodenhausen, G. V.; Macrae, C. y Sherman, J. (1999). "On the dialectics of discrimination: dual processes in social stereotyping». En: CHAIKEN, S. y Trope, Y. (eds.). Dual process theories in social psychology. Nueva York: Guilford.

BONNOT, V. y CROIZET, J. C. (2007). «Stereotype internalization and women's math performance: The role of interference in working memory». Journal of Experimental Social Psychology, 43, 857-866.

Briñol, P.; Falces, C. y Becerra, A. (2007). «Actitudes». En: Morales, J. F.; Huici, C.; Moya, M. y Gaviria, E. (eds.). Psicología social. Madrid: McGraw-Hill.

Cabré, A.; Domingo, A. y Menacho, T. (2002). «Demografía y crecimiento de la población española durante el siglo XX». En: Pimentel, M. (coord.). Mediterráneo Económico. Almería: Caja Rural Intermediterránea. Cajamar.

Cen D’Ancona, Ma . A. (2009). «La compleja detección del racismo y la xenofobia a través de encuesta. Un paso adelante en su medición». Revista Española de Investigaciones Sociológicas, 125, 13-45.

Cea d’Ancona, Ma. A. y Vallés Martínez, M. S. (2008). Evolución del racismo y la xenofobia en España [Informe 2008]. Madrid: OBERAXE. Ministerio de Trabajo e Inmigración.

- (2009). Evolución del racismo y la xenofobia en España [Informe 2009]. Madrid: OBERAXE. Ministerio de Trabajo e Inmigración.

- (2010). Evolución del racismo y la xenofobia en España [Informe 2010]. Madrid: OBERAXE. Ministerio de Trabajo e Inmigración.

Centro de Investigaciones Sociológicas (1995). Actitudes hacia la inmigración. Estudio 2131. Madrid: CIS.

- (2008). Actitudes hacia la inmigración (II). Estudio 2773. Madrid: CIS.

- (2009). Actitudes hacia la inmigración (III). Estudio 2817. Madrid: CIS.

Colectivo IOÉ (Pereda, C.; Actis, W. y Prada, M. A. de) (1995). Discurso de los españoles sobre los extranjeros. Paradojas de la alteridad. Madrid: CIS. Colección Opiniones y Actitudes, 8.

Colectivo IOÉ (Pereda, C.; Prada, M. A.; Actis, W. y Ortí, M.) (2010). Discursos de la población migrante en torno a su instalación en España. Exploración cualitativa. Madrid: CIS. Colección Opiniones y Actitudes, 64.

COlectivo IOÉ y OrTí, A. (2007). La convivencia en Madrid: Discursos ante el modelo de desarrollo de la ciudad y la instalación de población inmigrante. Madrid: Observatorio de las Migraciones y de la Convivencia Intercultural de la ciudad de Madrid.

COOPER, J. y FAZIO, R. H. (1984). "A new look in dissonance theory». En: BERKOWITZ, L. (eds.). Advances in experimental social psychology. San Diego, CA: Academic Press.

Czopp, A. M.; Monteith, M. J. y Mark, A. Y. (2006). «Standing up for a change: Reducing bias through interpersonal confrontation». Journal of Personality and Social Psychology, 90, 784-803.

Dasgupta, N. y GreEnWALD, A. G. (2001). "On the malleability of automatic attitudes: Combating automatic prejudice with images of admired and disliked individuals». Journal of Personality and Social Psychology, 81, 800-814.

Deutsch, M. y Gerard, H. B. (1955). "A study of normative and informational social influences upon individual judgment». Journal of Abnormal Social Psychology, 51, 629-636. 
Devine, P. G. (1989). «Stereotypes and prejudice: Their automatic and controlled components». Journal of Personality and Social Psychology, 56, 680-690.

DíEZ NiCOlÁs, J. (2009). «La construcción de un índice de Xenofobia-Racismo». Revista del Ministerio de Trabajo e Inmigración. Migraciones Internacionales, 80, 21-28.

Espelt, E.; Cornejo, J. M. y Javaloy, F. (2006). «La paradoja del racismo aversivo hacia los inmigrantes: un estudio experimental». Revista de Psicología Social, 21, 3-20.

Festinger, L. (1950). «Informal social communication». Psychological Review, 57, 271-282.

- (1957). A theory of cognitive dissonance. Stanford, CA: Stanford University Press.

Festinger, L.; SCHACHTER, S. y BACK, K. (1951). Social Pressure in informal groups. Nueva York: Harper.

FLOWERMAN, S. H. (1949). «The use of propaganda to reduce prejudice: A refutation». International Journal of Opinion and Attitude Research, 3, 99-108.

GAERTNER, S. L. y Dovidio, J. F. (1986). «The aversive form of racism». En: Dovidio, J. F. y Gaertner, S. L. (eds.). Prejudice, discrimination, and racism. Orlando, FL: Academic Press.

- (2000). Reducing intergroup bias: The Common Ingroup Identity Model. Filadelfia, PA: Psychology Press.

Gaertner, S.L.; Dovidio, J. F.; Rust, M. C.; Nier, J.; Banker, B. y Ward, C. M. (1999). "Reducing intergroup bias. Elements of intergroup cooperation». Journal of Personality and Social Psychology, 76, 388-402.

GARDINER, G. S. (1972). "Complexity training and prejudice reduction». Journal of Applied Social Psychology, 2, 326-342.

GÓmEZ, A. (2003). "Un ejemplo de estrategias para mejorar las relaciones entre grupos: la recategorización. Cómo hacer que diferentes grupos trabajen juntos en el mismo equipo». En: Morales, J. F. y Huici, C. (dirs.). Estudios de Psicología Social. Madrid: UNED.

- (2004). «La reducción del conflicto y la mejora de las relaciones intergrupales». En: Morales, J. F. y Huici, C. (dirs.). Psicología de grupos II. Métodos, técnicas y aplicaciones. Madrid: UNED.

Harding, J.; Proshansky, H. M.; Kutner, B. y Chein, I. (1969). «Prejudice and ethnic relations». En: Lindzey, G. y ARONSOn, E. (eds.). The handbook of social psychology. Reading, MA: Addison-Wesley.

Hewstone, M. y Brown, R. J. (1986). "Contact is not enough: An intergroup perspective on the Contact Hypothesis». En: Hewstone, M. y Brown, R. J. Contact and conflict in intergroup encounters. Oxford: Blackwell.

Hovland, C. I.; Lumsdaine, A. y Sheffield, F. (1949). Studies in social psychology in World War II. Vol. 3. Experiments on mass communication. Princeton, NJ: Princeton University Press.

Huici, C. (2008) «Las emociones intergrupales». En: Morales, J. F.; HuiCi, C.; Gaviria, E. y GÓmeZ, A. (eds.). Método, teoría e investigación en psicología social. Madrid: Pearson. Prentice Hall.

INNERARITY, C. y ACHA, B. (2010). «Los discursos sobre ciudadanía e inmigración en Europa: Universalismo, extremismo y educación». Política y Sociedad, 47 (2), 63-84.

JACKMAN, M. R. y CRANE, M. (1986). «Some of my best friends are black: Interracial friendship and whites' racial attitudes». Public Opinion Quarterly, 50, 459-486. 
JANIS, I. L. y KING, B. T. (1954). "The influence of role-playing on opinion change». Journal of Abnormal and Social Psychology, 49, 211-218.

Johnson, D. W. y Johnson, R. T. (2000). "The three Cs of reducing prejudice and discrimination». En: OSKAMP, S. (ed.). Reducing prejudice and discrimination. Hillsdale, NJ: Erlbaum.

JONES, E. E. (1990). Interpersonal perception. Nueva York: Freeman.

KaWAKami, K.; Dovidio, J. F. y VAN Kamp, S. (2005). «Kicking the habit: Effects of nonstereotypic association training and corrections processes on hiring decisions». Journal of Experimental Social Psychology, 41, 68-75.

Kunda, Z. y OlESON, K. C. (1995). «Maintaining stereotypes in the face of disconfirmation: Constructing grounds for subtyping deviants». Journal of Personality and Social Psychology, 68, 565-579.

LASSWELL, H. D. (1928). "The function of the propagandist». International Journal of Ethics, 38, 258-68.

LEIPPE, R. A. y EISENSTADT, D. (1994). "Generalization of dissonance reduction: Decreasing prejudice through induced compliance». Journal of Personality and Social Psychology, 67, 395-413.

LYNCH, J. (1986). Multicultural education: Principles and practice. Boston: Routledge y Kegan Paul.

Macrae, C. N.; Bodenhausen, G. V.; Milne, A. B. y Jetten, J. (1994). «Out of mind but back in sight: Stereotypes on the rebound». Journal of Personality and Social Psychology, 67, 808-817.

MAIO, G. R. y EsSES, V. (1998). "The social consequences of affirmative action: Deleterious effects on the perceptions of groups». Personality and Social Psychology Bulletin, 24, 65-74.

MCGREgOR, J. (1993). «Effectiveness of role playing and antiracist teaching in reducing student prejudice». Journal of Educational Research, 86, 215-226.

Merton, R. K. (1948). "The self-fulfilling prophecy». Antioch Review, 8, 193-210.

MolinA, L. E. y WiTTIG, M. A. (2006). "Relative importance of contact conditions in explaining prejudice reduction in a classroom context: Separate and Equal?». Journal of Social Issues, 62, 489-509.

Monteith, M. J.; Zuwerink, J. R. y Devine, P. G. (1994). «Prejudice reduction: Classic challenges, contemporary approaches». En: Devine, P. G.; Hamilton, D. L. y Ostrom, T. M. (eds.). Social cognition: Impact on social psychology. San Diego, CA: Academic Press.

Monteith, M. J.; Sherman, J. W. y Devine, P. G. (1998). «Suppression as a stereotype control strategy». Personality and Social Psychology Review, 2, 63-82.

Morales, L.; Anduiza, E.; Rodríguez, E. y SAN MARTín, J. (2008). "Capital social, pautas identitarias y actitudes hacia "los otros": La incorporación cívica de la población de origen inmigrante en Barcelona y Madrid». Panorama Social, 8, $119-142$.

NAVAS, M. S. (2008). «La integración de los inmigrantes: un análisis desde el Modelo Ampliado de Aculturación Relativa (MAAR)». En: Morales, J. F.; Huici, C.; GÓmeZ, A. y GAVIRIA, E. (coords.). Método, teoría e investigación en psicología social. Madrid: Pearson.

NeUBERG, S. L. (1994). «Expectancy-confirmation processes in stereotype-tinged social encounters: The moderating role of social goals». En: ZANNA, M. P. y OlsON, J. M. (eds.). The psychology of prejudice: The Ontario Symposium. Hillsdale, NJ: Erlbaum. 
Observatorio Permanente de la Inmigración (2010). Anuario Estadístico de Inmigración 2009 [en línea]. Madrid: Ministerio del Interior. <http://extranjeros. meyss.es/es/ObservatorioPermanenteInmigracion/Anuarios/Anuario2009.html> [Consulta: 11 noviembre 2011].

- (2012). Extranjeros residentes en España. Principales resultados 2011 [en línea]. Madrid: Ministerio del Interior. <http://extranjeros.meyss.es/es/Estadisticas/ operaciones/con-certificado/201112/Tablas_PR_31122011.pdf> [Consulta: 11 noviembre 2011].

PALUCK, E. L. (2009). «Reducing intergroup prejudice and conflict using the media: A field experiment in Rwanda». Journal of Personality and Social Psychology, 96, 574-587.

PetTigrew, T., F. (1979). «The ultimate attribution error: Extending Allport's cognitive analysis of prejudice». Personality and Social Psychology Bulletin, 5, 461-477.

- (1998). «Intergroup contact theory». Annual Review of Psychology, 49, 65-85.

PetTigrew, T. F. y Tropp, L. R. (2006). «A meta-analytic test of intergroup contact theory». Journal of Personality and Social Psychology, 90, 751-783.

Rodríguez, A.; Betancor, V. y Delgado, N. (2009). «La norma social sobre la expresión del prejuicio explícito hacia diferentes grupos sociales». Revista de Psicología Social, 24, 17- 27.

Rosenthal, R. y JACOBSON, L. (1968). Pygmalion in the classroom: Teacher expectation and pupils' intellectual development. Nueva York: Holt, Rinehart and Winston.

Rudman, L. A. y GLICK, P. (2001). "Prescriptive gender stereotypes and backlash toward agentic women». Journal of Social Issues, 57, 743-762.

Russell, M. J. (1961). Study of a South African inter-racial neighbourhood. Durban, S. Afr: Univ. Natal.

SAssenberG, K. y Moskowitz, G. B. (2005). «Don't stereotype, think different. Overcoming automatic stereotype activation by mindset priming». Journal of Experimental Social Psychology, 41, 506-514.

Schaller, M.; AsP, C. H.; Rosell, M. C. y Heim, S. J. (1996). «Training in statistical reasoning inhibits the formation of erroneous group stereotypes». Personality and Social Psychology Bulletin, 22, 829-844.

SHERIF, M. y SHERIF, C. W. (1953). Groups in harmony and tension: An integration of studies on intergroup relations. Nueva York: Octagon Books.

Sherif, M.; Harvey, O. J.; White, B. J.; Hood, W. R. y Sherif, C. W. (1961). Intergroup conflict and cooperation: The Robbers Cave experiment. Norman, OK: University of Oklahoma Book Exchange.

Sherman, J. W.; Stroessner, S. J.; Conrey, F. R. y Azam, O. A. (2005). «Prejudice and stereotype maintenance processes: Attention, attribution, and individuation». Journal of Personality and Social Psychology, 89, 607-622.

Sinclair, S.; DunN, E. y Lowery, B. (2005). "The relationship between parental racial attitudes and children's implicit prejudice». Journal of Experimental Social Psychology, 41, 283-289.

Singhal, A.; Cody, M. J.; Rogers, E. M. y SABIDO, M. (eds.) (2004). Entertainmenteducation and social change: History, research, and practice. Mahwah, NJ: Lawrence Erlbaum.

SNYDER, M. (1992). "Motivational foundations of behavioral confirmation». En Zanna, V. (ed.). Advances in experimental social psychology. Orlando, FL: Academic Press. 
SolÉ, C.; Sordé, T.; Serradell, O. et al. (2011). «Cohesión social e inmigración: Aportaciones científicas y discursos políticos». Revista Internacional de Sociología, 69 (1), 9-32.

STANGOR, C.; SeChrist, G. B. y Jost, J. T. (2001). «Changing racial beliefs by providing consensus information». Personality and Social Psychological Bulletin, 27, 484-494.

STEPHAN, W. G. y STEPHAN, C. W. (1984). «The role of ignorance in intergroup relations». En: Miller, N. y Brewer, M. B. (eds.). Groups in Contact: The Psychology of Desegregation. Nueva York: Academnic Press.

STEPHAN, W. G. y FINLAY, K. (1999). "The role of empathy in improving intergroup relations». Journal of Social Issues, 55, 729-743.

Stephan, W. G.; Boniecki, K. A.; YBARRA, O. et al. (2002). "The role of threats in the racial attitudes of Blacks and Whites». Personality and Social Psychology Bulletin, 28, 1242-1254.

Sullivan, J. y Transue, J. (1999). «The psychological underpinnings of democracy: A selective review of research on political tolerance, interpersonal trust, and social capital». Annual Review of Psychology, 50, 625-650.

TAjFeL, H. y Turner, J. C. (1986). "The social identity theory of intergroup behavior». En: Worschel, S. y Austin, W. G. Psychology of intergroup relations. Chicago: Nelson-Hall.

Trope, Y. y Liberman, A. (1996). "Social hypothesis testing: Cognitive and motivational mechanisms». En: Higgings, E. T. y Kruglanski, A. W. (eds.). Social Psychology: Handbook of basic principles. Nueva York: Guilford Press.

TURNER, J. C. (1987). Rediscovering the social group: self-categorization theory. Oxford, UK: B. Blackwell.

United Nations. Department of Economic and Social Affairs. Population DIVISION (2009). Trends in International Migrant Stock: The 2008 Revision (United Nations database, POP/DB/MIG/Stock/Rev.2008) [en línea]. <http://esa.un.org/ migration> [Consulta: 10 octubre 2011].

VAllés, M. S.; CEA D’Ancona, Ma A. e IZquierdo, A. (1999). Las encuestas sobre inmigración en España y Europa. Madrid. IMSERSO.

VRIJ, A.; VAN SCHIE, E. y ChERrYMAN, J. (1996). «Reducing ethnic prejudice through public communication programs: A social-psychological perspective». The Journal of Psychology, 130, 413-420.

VRIJ, A. y SMITH, B. (1999). "Reducing ethnic prejudice by public campaigns: An evaluation of a present and new campaign». Journal of Community y Applied Social Psychology, 9, 195-215.

Watson, G. (1947). Action for unity. Nueva York: Harper.

WheEler, S. C.; Jarvis, W. B. y PeTTY, R. E. (2001). «Think unto others: The selfdestructive impact of negative racial stereotypes». Journal of Experimental Social Psychology, 37, 173-180.

Williams, R. M. (1947). The reduction of intergroup tensions. Nueva York: Social Science Research Council.

Yzerbyt, V.; COUll, A. y RoCher, S. (1999). «Fencing off the deviant: The role of cognitive resources in the maintenance of stereotypes». Journal of Personality and Social Psychology, 77, 449-462.

ZELINGS, R. y HENDRICKSON, G. (1933). «Racial attitudes of 200 sixth grade children». Sociology y Social Research, 18, 26-36.

ZuWerink, J. R. y Devine, P. G. (1996). «Attitude importance and resistance to persuasion: it's not just the thought that counts». Journal of Personality and Social Psychology, 70, 931-944. 